\title{
Djulis (Chenopodium formosanum) and Its Bioactive Compounds for Management of Hyperlipidemia and Hyperglycemia in High-Fat Diet-Fed Mice
}

\author{
Shih-Ying CHEN ${ }^{1, \#}$, Chin-Chen CHU ${ }^{2, \#}$, Yi-Chiang LIN ${ }^{1}$, Pin-Der DUH ${ }^{3, *}$ \\ ${ }^{1}$ Department of Health and Nutrition, Chia Nan University of Pharmacy and Science, Tainan, Taiwan, ROC \\ ${ }^{2}$ Department of Anesthesiology, Chi-Mei Medical Center, Tainan, Taiwan, ROC \\ ${ }^{3}$ Department of Food Science and Technology, Chia Nan University of Pharmacy and Science, Tainan, Taiwan, ROC \\ ${ }^{\#}$ These authors contributed equally to this work. \\ *Corresponding author: ipdduh@mail.cnu.edu.tw
}

Received April 04, 2019; Revised May 14, 2019; Accepted June 12, 2019

\begin{abstract}
The aim of this study was to investigate the effect of Djulis (Chenopodium formosaneum) and its bioactive compounds on hyperlipidemia and hyperglycemia in high-fat diet (HFD)-fed mice. The study indicated that, total cholesterol (TC), and triglyceride (TG) levels were significantly higher in HFD-fed mice than in those fed with ethanolic extract of Djulis (EECF) at different doses $(10,25,50 \mathrm{mg} / \mathrm{kg} \mathrm{bw})$, rutin $(5 \mathrm{mg} / \mathrm{kg} \mathrm{bw})$, betanin $(5 \mathrm{mg} / \mathrm{kg} \mathrm{bw})$ and quercetin $(5 \mathrm{mg} / \mathrm{kg} \mathrm{bw})$ throughout the 12-week period, indicating alleviation of hyperlipidemia by EECF and bioactive compounds groups. Moreover, plasma insulin was significantly higher in HFD-fed mice than those fed with EECF at different doses, rutin, betanin and quercetin. In addition, results of oral glucose tolerance test (OGTT) revealed improved glucose tolerance and ameliorated insulin response at 60 and $90 \mathrm{~min}$ in mice fed with EECF, rutin, betanin and quercetin. The findings of this study suggest EECF has beneficial effects on hyperlipidemia and hyperglycemia of obese mice induced with HFD, which can be partly attributed to the bioactive compounds present in Djulis.
\end{abstract}

Keywords: betanin, Djulis (Chenopodium formosaneum), hyperlipidemia, hyperglycemia, quercetin, rutin

Cite This Article: Shih-Ying CHEN, Chin-Chen CHU, Yi-Chiang LIN, and Pin-Der DUH, "Djulis (Chenopodium formosanum) and Its Bioactive Compounds for Management of Hyperlipidemia and Hyperglycemia in High-Fat Diet-Fed Mice." Journal of Food and Nutrition Research, vol. 7, no. 6 (2019): 452-457. doi: 10.12691/jfnr-7-6-7.

\section{Introduction}

Hyperlipidemia, also called dyslipidemia, is a symptom of elevated lipid levels in blood plasma. A high-fat diet (HFD) causes an imbalanced lipoprotein metabolism and leads to hyperlipidemia, characterized as high levels of serum triglyceride (TG), total cholesterol (TC), and a low ratio of high density lipoprotein to total cholesterol (HDL/TC), which contribute to the key risk factor for cardiovascular diseases (CVD) [1]. In addition, dietinduced obesity leads to insulin insensitivity, which is related to low-grade inflammation [2]. It is known that high fat diet induced significant accumulation of advanced glycation end-products (AGEs) in the plasma, liver, kidney and adipose tissue of mice [2]. Although hyperlipidemia and hyperglycemia can be controlled by pharmacological strategies, some drugs could lead to side effects [3]. Therefore, development of logical and effective strategies for lowering hyperlipidemia and hyperglycemia is an important global issue. In addition, there is growing evidence that a diet rich in plants containing phytochemicals is correlated with a lower risk of chronic disease. Some plants that are used as a staple food contain bioactive compounds and have functional properties for preventing human diseases. Therefore, the search for functional biomaterials from natural plant-based foods and products capable of regulating lipid metabolisms and having antihyperlipidemic and antihyperglycemic effects is of great concern for researchers.

Djulis (Chenopodium formosanum) is a native cereal plant cultivated by aboriginals in Taiwan. Djulis is also called "Hung $\mathrm{Li}$ " because of its bright red color, is considered "ruby of cereals" [4]. Many recent studies have reported that Djulis contains valuable nutrients and bioactive compounds, and its use as functional foods has received increasing attention. Djulis has been found to have antioxidant potential and protect the skin against UV-induced damage [5,6]. It has also proved to be a potential anti-diabetic functional food ingredient [7]. Previous studies by the authors reported Djulis and its bioactive compounds, rutin, kaempferol and betanin, demonstrated hepatoprotection $[8,9]$, antihypertension [10], and inhibition of adipogenesis [11]. Apparently, Djulis has marked biological activity due to its bioactive 
compounds. Moreover, given its significant biological effects, Djulis may also regulate against hyperlipidemia and hyperglycemia. However, no reports on the effectiveness of Djulis in regulating hyperlipidemia and hyperglycemia have been found so far. In addition, previous studies by the authors showed bioactive compounds present in Djulis have biological effects and phytochemical characteristics, or a combination of the two [11]. Therefore, this study aims to investigate the effect of Djulis and its bioactive compounds on hypolipidemia and hypoglycemia in mice fed with a high-fat diet.

\section{Materials and Methods}

\subsection{Materials}

Diet D12450J and Diet D12492 were obtained from Research Diets, Inc. (New Brunswick, NJ, USA). Betanin, rutin and quercetin were obtained from Sigma (St. Louis, MO, USA). All other chemicals were of analytical grade.

\subsection{Sample Preparation}

Djulis (Chenopodium formosanum), identified by Professor Yau-Lun Kuo of the Department of Forestry, National Pingtung University of Science and Technology, was purchased from Kullku Farm, Pingtung, Taiwan, and was ground to a fine powder before the extraction. The voucher specimen (No. CNU-101) was deposited in the Department of Food Science and Technology, Chia Nan University of Pharmacy and Science. The powder $(10 \mathrm{~g})$ was extracted with $50 \%$ ethanol $(100 \mathrm{~mL})$ and stirred for $16 \mathrm{~h}$, at room temperature $\left(27 \pm 1^{\circ} \mathrm{C}\right)$. The extract was filtered and the residue was re-extracted under the same conditions. The combined filtrates were evaporated to dryness for ethanol under vacuum and weighed to determine the yield of soluble constituents. The ethanolic extract of Djulis, abbreviated as EECF, was stored at $4^{\circ} \mathrm{C}$ until used [11].

\subsection{Animals and Experimental Design}

Five-week-old male C57BL/6 mice were purchased from National Laboratory Animal Center (Taipei, Taiwan). During the acclimatization period, each animal was raised on a commercial rodent chow diet (Lab Diet 5001) ad libitum. After a 1-week acclimation period, mice were divided randomly into two groups. The control group (CON) was fed low-fat (LF) diet (Diet 12450J, Research Diets) whereas the experimental group was fed a $60 \%$ high-fat diet (Diet 12492, Research Diets) for 12 weeks. After 8 week of induction, the CON was subdivided into two groups and was treated with vehicle only (RD) or EECF $(50 \mathrm{mg} / \mathrm{kg}$ bw, RD+HCF) 4 weeks afterward. The experimental group was subdivided into seven groups and was given orally with vehicle only (HFD), 10, 25, $50 \mathrm{mg}$ of EECF / kg bw (HFD+LCF, HFD+MCF, HFD+HCF), 5 $\mathrm{mg}$ of rutin / $\mathrm{kg}$ bw $(\mathrm{HFD}+\mathrm{R}), 5 \mathrm{mg}$ of betanin $/ \mathrm{kg}$ bw $(\mathrm{HFD}+\mathrm{B})$ and $5 \mathrm{mg}$ of quercetin / $\mathrm{kg}$ bw (HFD+Q) 4 weeks afterward, respectively. Animals were cared and used after the experimental protocols were approved by institutional animal ethics committee (Chia-Nan
University, Tainan, Taiwan). All animals were housed in individual cages with food and water ad libitum, weighed periodically and maintained in a controlled environment at $24 \pm 1{ }^{\circ} \mathrm{C}, 55 \pm 5 \%$ relative humidity and a cycle of $12 \mathrm{~h}$ dark/light in the entire experiment. At the end of 12 weeks of treatment, rats were asphyxiated by carbon dioxide and then blood was withdrawn from the heart (cardiac puncture) with a heparinized collection tube. Blood was centrifuged at $1,000 \times g$ for $10 \mathrm{~min}$ and plasma obtained was frozen at $-20{ }^{\circ} \mathrm{C}$ until analysis.

\subsection{Plasma Biochemical Assays}

The plasma triglyceride concentration was measured using commercially available kits (Triglycerides Liquid; Sentinel Diagnostics, Milan, Italy). Plasma cholesterol (TC), HDL-cholesterol (HDL-C) and LDL-cholesterol (LDL-C) concentrations were determined using commercially available enzymatic kits (T-CHO, HDL-EX(N) and LDL-EX(N); Denka Seiken, Tokyo, Japan). Plasma non-esterified fatty acid (NEFA) concentrations were assayed by an enzymatic colorimetric method (Randox NEFA kit FA115; Antrim, UK). Kits for alanine transaminase (ALT) and aspirate transaminase (AST) were obtained from Denka Seiken Co., Ltd. (Tokyo, Japan). These assays were completed according to methodology recommended by the manufacturer and detected by a biochemical autoanalyzer (Toshiba, TBA-200FR, Holliston, MA, USA). Plasma insulin and leptin levels were determined with ELISA immunoassay (Mercodia Mouse Insulin ELISA, Uppsala, Sweden, and Diagnostic Systems Laboratories, Inc, Texas USA, respectively).

\subsection{Oral glucose Tolerance Test}

An oral glucose tolerance test (OGTT) was performed at the end of the treatment. On the test day, animals were fasted for $10 \mathrm{~h}$, and glucose $(1.5 \mathrm{~g} / \mathrm{kg})$ was then orally administered to them. Plasma glucose levels were determined from the tail vein at 0 (before glucose challenge), 30, 60, and $90 \mathrm{~min}$ after glucose administration. Glucose colorimetric assay kit was purchased from Cayman Chemical Co. (MI, USA). During OGTT at 15, 30, 60 and 90 minutes blood was drawn for glucose and insulin, allowing calculations area under the curve (AUC) and homeostasis model assessment (HOMA) [12].

\subsection{Statistical Analyses}

Data are expressed as mean \pm SEM, and ANOVA was conducted by using the SPSS software (version 12.0; SPSS, Chicago, IL, USA). When a significant $\mathrm{F}$ ratio was obtained $(p<0.05)$ a post hoc analysis was conducted between groups by using a multiple comparison procedure with a Duncan's multiple range test. $P$ values $<0.05$ were considered significant.

\section{Results \& Discussion}

In a previous study by the authors [11], rutin, quercetin derivatives, betanin and other compounds were present in EECF using HPLC-DAD and HPLC-MS/MS analysis. 
Among those compounds identified, rutin and quercetin derivatives are the two most abundant compounds in EECF. In addition, betanin represents a group of pigments responsible for the red color in Djulis [4]. Therefore, rutin, quercetin and betanin were selected as reference compounds for the present series of in vivo experiments.

\subsection{Effects of EECF on the Body Weight, Food Intake and Feed Efficiency}

As shown in Table 1, the final body weight, daily food intake and feed efficiency of the HFD group were higher than those in the control group (RD and RD+HCF, $p<0.05$ ). The mice fed with HFD plus EECF at different concentrations or betanin showed no significant differences in daily weight gain, daily food intake and feed efficiency, compared with mice fed with HFD alone (Table 1), indicating co-feeding EECF at different concentrations and betanin with HFD which had no effect on body weight gain.

\subsection{Effects of EECF on Plasma Lipid Levels}

Table 2 shows the effects of EECF on plasma lipid levels of mice fed with HFD. The plasma in HFD-fed mice showed significant increments in TG, TC and HDL-C, compared with the control group, whereas supplementation with EECF at $10,25,50 \mathrm{mg} / \mathrm{kg}$ bw, and $5 \mathrm{mg} / \mathrm{kg}$ bw rutin, betanin and quercetin decreased markedly both TG and TC levels when compared with those in mice fed with HDF alone. No significant differences in LDL-C and NEFA were observed in mice fed with HFD plus EECF at different concentrations, rutin, betanin and quercetin. HDL-C levels were significantly higher in HFD-fed mice than in those fed with EECF at $50 \mathrm{mg} / \mathrm{kg}$ bw. These results show EECF and its bioactive compounds, rutin, betanin and quercetin, could remarkably improve lipid profiling in HFD-fed mice. From the perspective of therapy, EECF, rutin, quercetin and betanin could prevent dyslipidemia.

Table 1. Effects of EECF and Its Bioactive Compounds on Body Weight of Mice Fed with HFD

\begin{tabular}{|c|c|c|c|c|c|}
\hline Groups & Initial weight (g) & Final body weight (g) & Daily weight gain (g/day) & Daily food intake (g/day) & Feed efficiency $(\%)$ \\
\hline RD & $21.48 \pm 0.51$ & $28.00 \pm 1.62^{\mathrm{a}}$ & $0.08 \pm 0.01$ & $3.12 \pm 0.23^{\mathrm{d}}$ & $2.55 \pm 0.17^{\mathrm{a}}$ \\
\hline $\mathrm{RD}+\mathrm{HCF}$ & $21.48 \pm 0.22$ & $27.95 \pm 1.56^{\mathrm{a}}$ & $0.08 \pm 0.01$ & $3.07 \pm 0.05^{\mathrm{d}}$ & $2.58 \pm 0.27^{\mathrm{a}}$ \\
\hline HFD & $22.66 \pm 0.58$ & $33.12 \pm 1.73^{\mathrm{b}}$ & $0.13 \pm 0.03$ & $2.82 \pm 0.02^{\mathrm{bc}}$ & $4.61 \pm 1.03^{\mathrm{b}}$ \\
\hline $\mathrm{HFD}+\mathrm{LCF}$ & $22.08 \pm 0.27$ & $31.56 \pm 0.97^{\mathrm{ab}}$ & $0.11 \pm 0.01$ & $2.80 \pm 0.02^{\mathrm{bc}}$ & $3.76 \pm 0.41^{\mathrm{ab}}$ \\
\hline $\mathrm{HFD}+\mathrm{MCF}$ & $22.05 \pm 0.15$ & $31.34 \pm 1.18^{\mathrm{ab}}$ & $0.10 \pm 0.02$ & $2.77 \pm 0.02^{\mathrm{ab}}$ & $3.77 \pm 0.59^{\mathrm{ab}}$ \\
\hline $\mathrm{HFD}+\mathrm{HCF}$ & $22.20 \pm 0.57$ & $30.22 \pm 1.39^{\mathrm{ab}}$ & $0.09 \pm 0.02$ & $2.83 \pm 0.02^{\mathrm{c}}$ & $3.31 \pm 0.77^{\mathrm{ab}}$ \\
\hline HFD+R & $21.84 \pm 0.48$ & $28.51 \pm 1.55^{\mathrm{a}}$ & $0.10 \pm 0.01$ & $2.73 \pm 0.01^{\mathrm{a}}$ & $3.68 \pm 0.31^{\mathrm{ab}}$ \\
\hline $\mathrm{HFD}+\mathrm{Q}$ & $22.17 \pm 0.78$ & $28.21 \pm 1.93^{\mathrm{a}}$ & $0.08 \pm 0.04$ & $2.82 \pm 0.03^{\mathrm{bc}}$ & $2.71 \pm 1.24^{\mathrm{ab}}$ \\
\hline
\end{tabular}

RD: low-fat diet; RD+HCF: high dosage of EECF with RD; HFD: high-fat diet; LCF: low dosage of EECF; MCF: medium dosage of EECF; HCF: high dosage of EECF; R: rutin; B: betanin; Q: quercetin. Values represent the mean \pm SEM $(n=6)$. Statistical methods for high fat diet groups are used by Duncan's multiple range test, the different superscript letters ${ }^{(\mathrm{a}-\mathrm{b})}$ are significantly different at $p<0.05$. Feed efficiency $(\%)=($ daily weight gain $/$ daily food intake) $\times 100$.

Table 2. Effects of EECF on the Plasma Lipid Levels of Mice Fed with HFD

\begin{tabular}{|c|c|c|c|c|c|}
\hline Groups & $\begin{array}{c}\text { TG } \\
(\mathrm{mg} / \mathrm{dL})\end{array}$ & $\begin{array}{c}\mathrm{TC} \\
(\mathrm{mg} / \mathrm{dL}) \\
\end{array}$ & $\begin{array}{l}\text { HDL-C } \\
(\mathrm{mg} / \mathrm{dL}) \\
\end{array}$ & $\begin{array}{l}\text { LDL-C } \\
(\mathrm{mg} / \mathrm{dL})\end{array}$ & $\begin{array}{c}\text { NEFA } \\
(\mathrm{mmol} / \mathrm{L})\end{array}$ \\
\hline $\mathrm{RD}$ & $32.40 \pm 6.14^{\mathrm{a}}$ & $136.60 \pm 3.26^{\mathrm{a}}$ & $113.20 \pm 2.87^{\mathrm{a}}$ & $17.00 \pm 1.29$ & $1.17 \pm 0.11^{\mathrm{a}}$ \\
\hline $\mathrm{RD}+\mathrm{HCF}$ & $49.57 \pm 6.14^{\mathrm{ab}}$ & $142.60 \pm 4.02^{\mathrm{a}}$ & $115.80 \pm 4.16^{\mathrm{ab}}$ & $17.29 \pm 1.91$ & $1.42 \pm 0.06^{\mathrm{ab}}$ \\
\hline HFD & $139.00 \pm 18.77^{\mathrm{c}}$ & $209.67 \pm 40.93^{\mathrm{b}}$ & $141.33 \pm 2.33^{\mathrm{cd}}$ & $30.66 \pm 16.92$ & $2.00 \pm 0.32^{\mathrm{b}}$ \\
\hline $\mathrm{HFD}+\mathrm{LCF}$ & $83.75 \pm 15.93^{\mathrm{b}}$ & $152.80 \pm 4.22^{\mathrm{a}}$ & $124.00 \pm 3.03^{\mathrm{abc}}$ & $17.20 \pm 2.44$ & $1.56 \pm 0.19^{\mathrm{ab}}$ \\
\hline HFD+MCF & $82.40 \pm 15.27^{b}$ & $135.60 \pm 13.53^{\mathrm{a}}$ & $127.50 \pm 2.96^{\mathrm{bcd}}$ & $14.83 \pm 1.85$ & $1.61 \pm 0.32^{\mathrm{ab}}$ \\
\hline $\mathrm{HFD}+\mathrm{HCF}$ & $82.75 \pm 15.15^{b}$ & $139.20 \pm 4.24^{\mathrm{a}}$ & $118.33 \pm 3.55^{\mathrm{ab}}$ & $13.00 \pm 1.93$ & $1.51 \pm 0.09^{\mathrm{ab}}$ \\
\hline $\mathrm{HFD}+\mathrm{R}$ & $67.67 \pm 3.18^{\mathrm{ab}}$ & $140.00 \pm 25.54^{\mathrm{a}}$ & $140.75 \pm 2.87^{\mathrm{d}}$ & $14.75 \pm 3.71$ & $1.39 \pm 0.11^{\mathrm{ab}}$ \\
\hline HFD+B & $51.60 \pm 5.57^{\mathrm{ab}}$ & $145.40 \pm 6.87^{\mathrm{a}}$ & $121.20 \pm 5.60^{\mathrm{abc}}$ & $19.17 \pm 2.70$ & $1.59 \pm 0.09^{\mathrm{ab}}$ \\
\hline $\mathrm{HFD}+\mathrm{Q}$ & $46.75 \pm 12.93^{\mathrm{ab}}$ & $158.40 \pm 8.75^{\mathrm{a}}$ & $127.50 \pm 5.93^{\mathrm{bcd}}$ & $17.00 \pm 2.81$ & $1.49 \pm 0.12^{\mathrm{ab}}$ \\
\hline
\end{tabular}

RD: low-fat diet; RD+HCF: high dosage of EECF with RD; HFD: high-fat diet; LCF: low dosage of EECF; MCF: medium dosage of EECF; HCF: high dosage of EECF; R: rutin; B: betanin; Q: quercetin. Values represent the mean \pm SEM $(n=6)$. Statistical methods for high fat diet groups are used by Duncan's multiple range test, the different superscript letters ${ }^{(\mathrm{a}-\mathrm{b})}$ are significantly different at $p<0.05$. TC, total cholesterol; TG, triglyceride; HDL-C, high density lipoprotein cholesterol; LDL-C, low density lipoprotein cholesterol; NEFA, non-esterified fatty acids.

Table 3. Effects of EECF and Its Bioactive Compounds on Biochemical Examination for the Plasma of Mice Fed with HFD

\begin{tabular}{|c|c|c|c|c|}
\hline Groups & AST (U/L) & ALT (U/L) & Insulin $(\mu \mathrm{g} / \mathrm{L})$ & Leptin $(n \mathrm{~g} / \mathrm{L})$ \\
\hline $\mathrm{RD}$ & $204.25 \pm 39.67$ & $37.40 \pm 7.93$ & $0.81 \pm 0.11^{\mathrm{a}}$ & $12.04 \pm 3.22^{\mathrm{a}}$ \\
\hline $\mathrm{RD}+\mathrm{HCF}$ & $215.75 \pm 7.49$ & $43.40 \pm 13.12$ & $0.66 \pm 0.02^{\mathrm{a}}$ & $15.77 \pm 2.76^{\mathrm{ab}}$ \\
\hline HFD & $208.20 \pm 22.92$ & $47.60 \pm 12.65$ & $2.00 \pm 0.48^{\mathrm{b}}$ & $62.80 \pm 8.77^{\mathrm{cd}}$ \\
\hline $\mathrm{HCD}+\mathrm{LCF}$ & $136.00 \pm 50.50$ & $24.25 \pm 5.27$ & $0.79 \pm 0.15^{\mathrm{a}}$ & $50.29 \pm 11.28^{\mathrm{bc}}$ \\
\hline HFD+MCF & $214.25 \pm 35.95$ & $40.40 \pm 8.33$ & $0.91 \pm 0.14^{\mathrm{a}}$ & $43.68 \pm 10.02^{\mathrm{abc}}$ \\
\hline $\mathrm{HFD}+\mathrm{HCF}$ & $143.50 \pm 20.60$ & $28.60 \pm 6.78$ & $0.87 \pm 0.15^{\mathrm{a}}$ & $44.64 \pm 9.53^{\mathrm{abc}}$ \\
\hline HFD+R & $161.00 \pm 55.52$ & $30.75 \pm 6.05$ & $0.81 \pm 0.02^{\mathrm{a}}$ & $92.89 \pm 19.14^{\mathrm{d}}$ \\
\hline HFD+B & $176.50 \pm 3.80$ & $44.00 \pm 14.37$ & $1.00 \pm 0.31^{\mathrm{a}}$ & $52.06 \pm 21.40^{\mathrm{bc}}$ \\
\hline $\mathrm{HFD}+\mathrm{Q}$ & $162.50 \pm 31.42$ & $21.25 \pm 2.02$ & $0.83 \pm 0.05^{\mathrm{a}}$ & $50.31 \pm 11.48^{\mathrm{bc}}$ \\
\hline
\end{tabular}

RD: low-fat diet; RD+HCF: high dosage of EECF with RD; HFD: high-fat diet; LCF: low dosage of EECF; MCF: medium dosage of EECF; HCF: high dosage of EECF; R: rutin; B: betanin; Q: quercetin. Values represent the mean \pm SEM $(n=6)$. Statistical methods for high fat diet groups are used by Duncan's multiple range test, the different superscript letters ${ }^{(\mathrm{a}-\mathrm{b})}$ are significantly different at $p<0.05$. AST, aspartate aminotransferase; ALT, alanine aminotransferase. 


\subsection{Effect of EECF on Plasma Biochemical Markers}

Table 3 shows the effects of EECF and its bioactive compounds, rutin, betanin and quercetin, on plasma biochemical markers in HFD-fed mice. Although no significant differences in AST and ALT levels were observed between any of the dietary groups, mice treated with EECF at different concentrations, rutin, betanin and quercetin tended to have lower ALT and AST activities, compared with the HFD group. These results suggest the occurrence of a compensatory hepatic response, which lessened HFD-induced liver injury [13]. This probably explains why EECF and its bioactive compounds did not show any significant positive effect on AST and ALT activities [4]. Table 3 also shows the plasma insulin and leptin levels of mice in each group. As can be seen, the insulin levels were higher in HFD-fed mice than in the control group, evidencing that long-term elevation of fatty acids from HFD raises plasma insulin levels by stimulating insulin secretion or by decreasing insulin clearance [14]. Meanwhile, plasma insulin levels were significantly lower in HFD-fed mice treated with EECF at different concentrations, rutin, betanin and quercetin than non-treated mice, implying that EECF could effectively reduce insulin in mice fed with HFD. In addition, plasma leptin levels were significantly higher in the HFD group than the control groups. There were no significant differences among mice fed with EECF at different concentrations, rutin, betanin and quercetin, but these mice tended to have lower leptin compared with mice fed with HFD alone, except rutin. Leptin is an adipocyte-secreted hormone that plays an important role in energy homeostasis. However, increase in plasma leptin level is associated with obesity induced by HFD [15].

As seen in Table 3, increased leptin levels were observed after HFD intake, while the pretreatment of EECF, betanin and quercetin tended to prevent increasing leptin levels in HFD-fed mice.

\subsection{Effect of the EECF on Glucose Tolerance}

To determine whether glucose levels in mice fed with
HFD are affected by EECF and its bioactive compounds, an oral glucose tolerance test (OGTT) was done. As shown in Figure 1, plasma glucose levels increased after the start of HFD, compared with the control group. The plasma glucose levels increased with increasing time points up to $30 \mathrm{~min}$, and then decreased with increasing time. Lower plasma glucose levels were found in mice given EECF at different concentrations, rutin, betanin and quercetin, especially at the 60 -min time point. In addition, the area under the curve (AUC) for all the groups was determined. As seen in Figure 2, EECF at 10, 25, $50 \mathrm{mg} / \mathrm{kg}$ bw and rutin, betanin, quercetin at $5 \mathrm{mg} / \mathrm{kg} \mathrm{bw}$ showed a $19.2 \%, 15.8 \%, 19.2 \%, 16.7 \%, 19.3 \%, 20.6 \%$ reduction in AUC, respectively, compared with mice fed with a HFD alone. Glucose tolerance after 4 weeks on diet showed similar AUC for the groups fed with EECF at different concentrations, rutin, betanin and quercetin, which were all lower than that for the HFD group. These results suggest improved glucose tolerance following the administration of EECF, rutin, betanin and quercetin. Insulin resistance was evaluated using homeostatic model assessment (HOMA). Figure 3 shows HOMA-IR in mice fed with HFD and those treated with EECF at different concentrations, rutin, betanin, and quercetin. As can be seen, the HOMA-IR index in HFD group increased by $116 \%$ compared with the control group, revealing clearly the development of insulin resistance in the HFD group [16]. However, the mice treated with EECF, rutin, betanin, and quercetin showed lower HOMA-IR values than the HFD group, indicating inverse association between administration of EECF, rutin, betanin and quercetin and HOMA-IR in mice. Several studies have noted that lipids could induce insulin resistance by interfering with downstream signal transduction of the insulin receptor [16]. The improvement of glucose homeostasis observed in this study could be associated with the low glucose uptake induced by EECF, rutin, betanin and quercetin, compared with the HFD group [17]. As a consequence of low blood glucose levels, the pancreatic secretory capacity could be reduced, thereby producing low insulin levels, as confirmed by low HOMA-IR index and insulin levels obtained in EECF, rutin, betanin and quercetin groups [17].

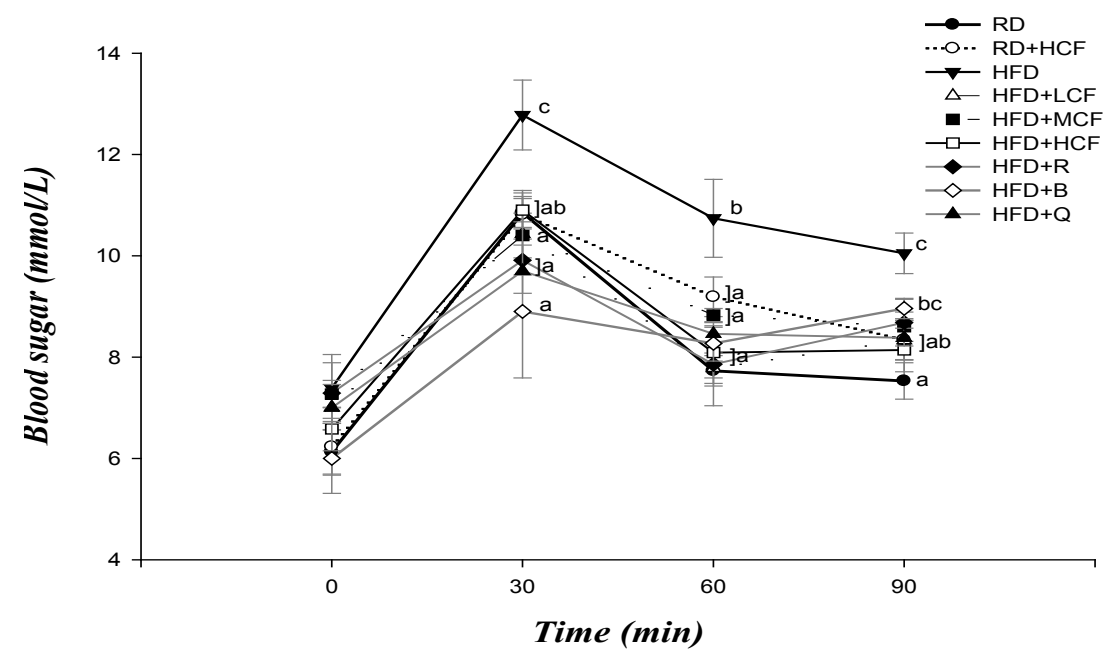

Figure 1. Plasma glucose responses to an oral glucose challenge $(1.5 \mathrm{~g} / \mathrm{kg})$ after $12 \mathrm{~h}$ of food deprivation at day 84 . RD: low-fat diet; RD+HCF: high dosage of EECF with RD; HFD: high-fat diet; LCF: low dosage of EECF; MCF: medium dosage of EECF; HCF: high dosage of EECF; R: rutin; B: betanin; Q: quercetin. Values represent the mean \pm SEM $(n=6)$. Statistical methods for high fat diet groups are used by Duncan's multiple range test, the different superscript letters ${ }^{(\mathrm{a}-\mathrm{b})}$ are significantly different at $p<0.05$ 


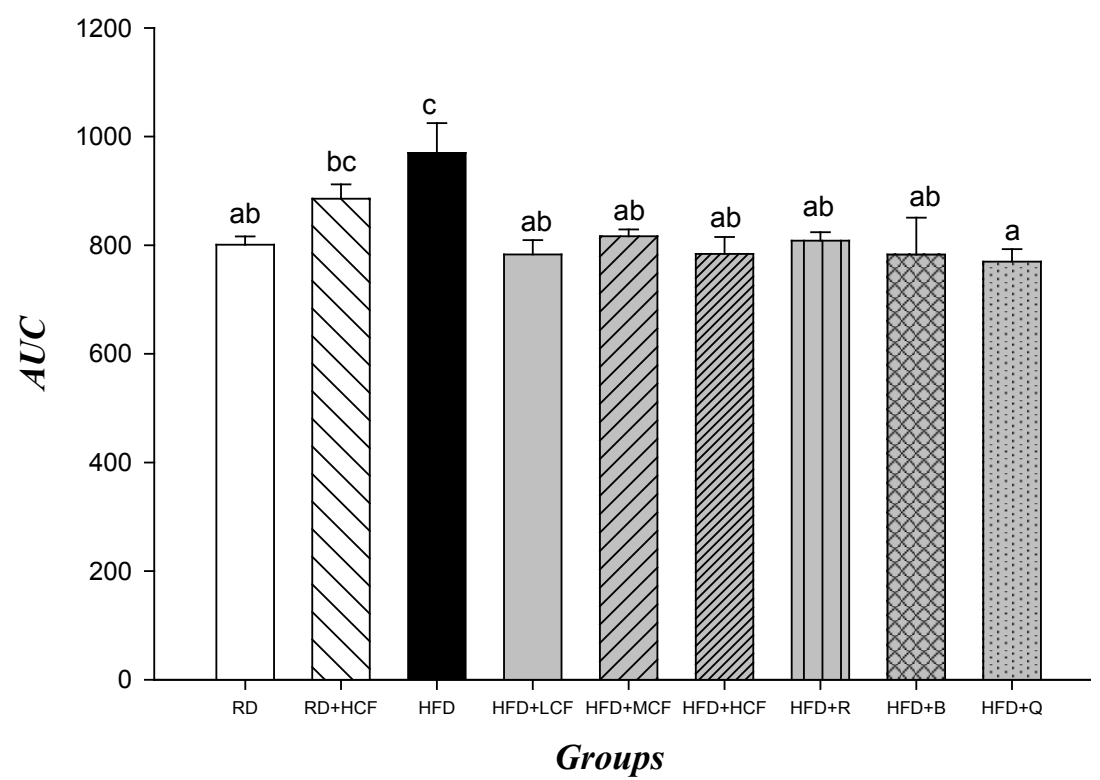

Figure 2. Effects of test samples on area under the curve (AUC) of glucose levels from oral glucose tolerance test (OGTT) for C57BL/6 mice fed with high fat diet. RD: low-fat diet; RD+HCF: high dosage of EECF with control diet; HF: high-fat diet; LCF: low dosage of EECF; MCF: medium dosage of EECF; HCF: high dosage of EECF; R: rutin; B: betanin; Q: quercetin. Values represent the mean \pm SEM $(n=6)$. Statistical methods for high fat diet groups are used by Duncan's multiple range test, the different superscript letters ${ }^{(\mathrm{a}-\mathrm{b})}$ are significantly different at $p<0.05$. AUC: The area under the curve (AUC) of glucose levels.

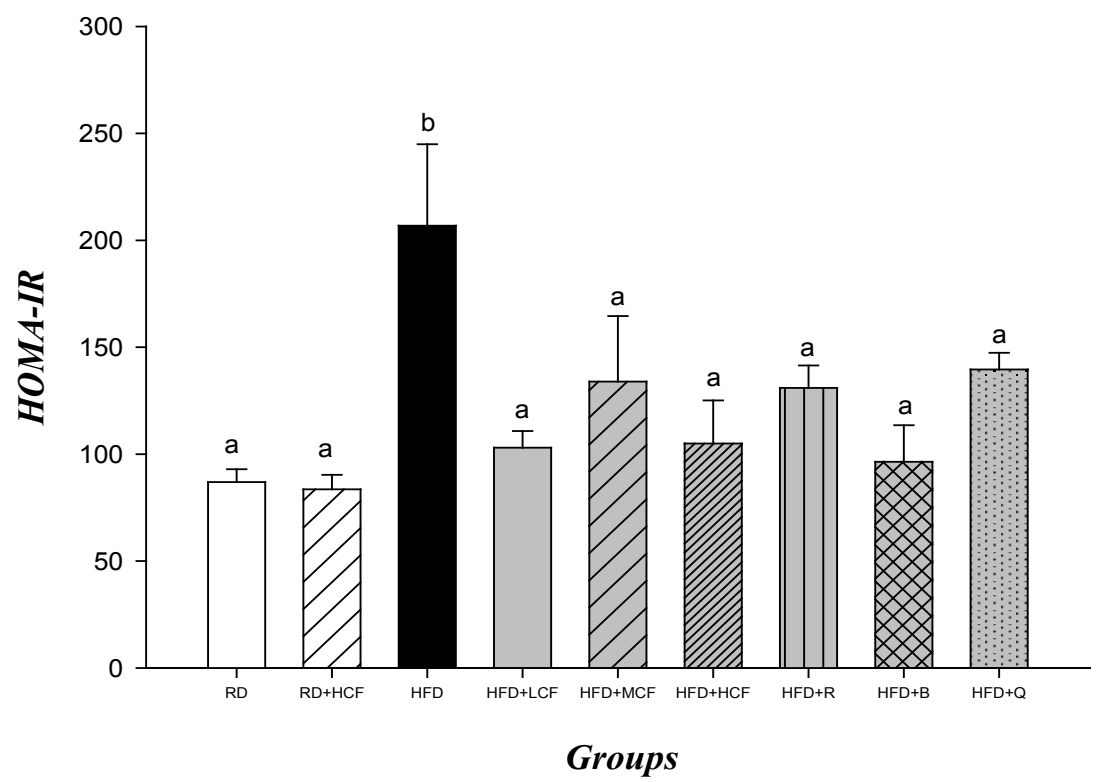

Figure 3. Effects of ethanolic extracts of Djulis (Chenopodium Formosanum) (EECF) and its bioactive compounds including rutin, quercetin and betanin on homeostasis model assessment (HOMA-IR) of C57BL/6 mice fed with high fat diet. RD: low-fat diet; RD+HCF: high dosage of EECF with control diet; HF: high-fat diet; LCF: low dosage of EECF; MCF: medium dosage of EECF; HCF: high dosage of EECF; R: rutin; B: betanin; Q: quercetin. Values represent the mean \pm SEM $(n=6)$. Statistical methods for high fat diet groups are used by Duncan's multiple range test, the different superscript letters ${ }^{(\mathrm{a}-\mathrm{b})}$ are significantly different at $p<0.05$. HOMA-IR index $=$ insulin $(\mathrm{mU} / \mathrm{L}) \times \mathrm{glucose}(\mathrm{mmol} / \mathrm{L}) / 22.5$

Phytochemicals have been proven to show biological activities. In this study, rutin, betanin and quercetin showed significant antihyperlipidemic effect on HFD-fed mice. These findings are in agreement with the report that rutin not only suppressed oleic-induced lipid accumulation in hepatocytes but also attenuated the HFD-induced metabolic syndrome. In the previous study by the authors showed that the suppression of lipid accumulation by rutin and betanin occurred through decreasing the gene expression of PPAR $\gamma, \mathrm{C} / \mathrm{EBP} \alpha$ and SREBP-1c [11]. In addition, high-fat and sucrose-fed obesity-induced rats treated with quercetin showed significantly lower blood glucose levels than control rats [18]. Both decreases in plasma glucose and increase in insulin levels were observed along with the restoration of glycogen content and the activities of carbohydrate metabolic enzymes in rutin-treated diabetic rats [19]. Betanin reveals antidiabetic properties by controlling the activities of liver enzymes [20,21]. Collectively, these results indicate that rutin, quercetin, betanin present in EECF may in part account for the antihyperlipidemic and antihyperglycemic effects of EECF. In addition, there are other peaks present in EECF besides rutin, betanin and quercetin, indicating the presence of other uncharacterized bioactive compounds in EECF [11]. These compounds may demonstrate biological activity due to the synergy between compounds, thereby 
contributing to antihyperlipidemic and antihyperglycemic effects of EECF in HFD-fed mice.

\section{Conclusion}

In conclusion, the present findings support the view that EECF may regulate parameters that are altered in the metabolic syndrome such as blood lipids, glycemia and insulinemia. These results obtained suggest that EECF could be a nutraceutical agent and functional food for ameliorating the metabolic syndrome induced by highfat diet. The antihyperlipidemic and antihyperglycemic effects of EECF are through effective modulation by its active compounds. Although the antihyperlipidemic and antihyperglycemic profiles of mice are different from those of humans, these results contribute to a better understanding of the effects of EECF on metabolic syndrome. Thus, regular Djulis consumption has beneficial effects against hyperlipidemia and hyperglycemia.

\section{Conflict of Interest}

The authors declare no conflict of interest.

\section{Acknowledgements}

This research study was supported by research grants from the National Science Council of the Republic of China NSC 102-2313-B-041-001-MY3 and Chi Mei Medical Center (CMFHR10205).

\section{References}

[1] L. K. Liu, F. P. Chou, Y. C. Chen, C. C. Chyau, H. H. Ho, and C. J. Wang, "Effects of mulberry (Morus alba L.) extracts on lipid homeostasis in vitro and in vivo," J. Agric. Food Chem., vol. 57, no. 16, pp. 7605-7611, 2009.

[2] L. M. Williams, F. M. Campbell, J. E. Drew, C. Koch, N. Hoggard, W. D. Rees, T. Kamolrat, H. Thi Ngo, I. L. Steffensen, S. R. Gray, and A. Tups, "The development of diet-induced obesity and glucose intolerance in C57BL/6 mice on a high-fat diet consists of distinct phases," PloS one, vol. 9, no. 8, pp. e106159, 2014.

[3] H. Xu, C. F. Zhao, G. G. Tian, L. R. Qian, and L. J. Yu, "Characterization of active constituents in Pyracantha fortuneana fruit extract and their effects on hyperlipidaemia, obesity, and oxidative stress in rodents," J. Funct. Foods, vol. 22, pp. 278-290, 2016.

[4] S. Y. Chen, C. C. Chu, C. C. Chyau, Z. H. Fu, and P. D. Duh, "Effect of water extract of Djulis (Chenopodium formosaneum) and its bioactive compounds on alcohol-induced liver damage in rats," Int. J. Food Sci. Nutr., vol. 5, no. 1, pp. 55-63, 2018.

[5] Y. H. Hong, Y. L. Huang, Y. C. Liu, and P. J. Tsai, "Djulis (Chenopodium formosanum Koidz.) water extract and its bioactive components ameliorate dermal damage in UVB-irradiated skin models," Biomed. Res. Int., vol. 2016, 2016.
[6] P. J. Tsai, Y. S. Chen, C. H. Sheu, and C. Y. Chen, "Effect of nanogrinding on the pigment and bioactivity of djulis (Chenopodium formosanum Koidz.)," J. Agric. Food Chem., vol. 59, no. 5, pp. 1814-1820, 2011.

[7] B. Y. Hsu, S. Y. Pan, L. Y. Wu, C. T. Ho, and L. S. Hwang, "Hypoglycemic activity of Chenopodium formosanum Koidz. components using a glucose uptake assay with 3T3-L1 adipocytes," Food Biosci., vol. 24, pp. 9-16, 2018.

[8] C. C. Chyau, C. C. Chu, S. Y. Chen, and P. D. Duh, "Djulis (Chenopodiun formosaneum) and its bioactive compounds protect against oxidative stress in human HepG2 cells," J. Funct. Foods, vol. 18, pp. 159-170, 2015.

[9] C. C. Chu, S. Y. Chen, C. C. Chyau, Z. H. Fu, C. C. Liu, and P. D. Duh, "Protective effect of Djulis (Chenopodium formosanum) and its bioactive compounds against carbon tetrachloride-induced liver injury, in vivo," J. Funct. Foods, vol. 26, pp. 585-597, 2016.

[10] S. Y. Chen, C. C. Chub, C. C. Chyau, J. W. Yang, and P. D. Duh, "Djulis (Chenopodium formosanum) and its bioactive compounds affect vasodilation, angiotensin converting enzyme activity, and hypertension," Food Biosci., vol. submitted, 2019.

[11] C. C. Chyau, C. C. Chu, S. Y. Chen, and P. D. Duh, "The inhibitory effects of djulis (Chenopodium formosanum) and its bioactive compounds on adipogenesis in 3T3-L1 adipocytes," Molecules, vol. 23, no. 7, pp. 1780, 2018.

[12] P. Zabielski, H. R. Hady, M. Chacinska, K. Roszczyc, J. Gorski, and A. U. Blachnio-Zabielska, "The effect of high fat diet and metformin treatment on liver lipids accumulation and their impact on insulin action," Sci. Rep., vol. 8, no. 1, pp. 7249, 2018.

[13] W. C. Chiu, Y. L. Huang, Y. L. Chen, H. C. Peng, W. H. Liao, H. L. Chuang, J. R. Chen, and S. C. Yang, "Synbiotics reduce ethanol-induced hepatic steatosis and inflammation by improving intestinal permeability and microbiota in rats," Food Funct., vol. 6, no. 5, pp. 1692-1700, 2015.

[14] G. Boden, "Free fatty acids and insulin secretion in humans," Curr. Diab. Rep., vol. 5, no. 3, pp. 167-170, 2005.

[15] L. Jiang, Q. Wang, Y. Yu, F. Zhao, P. Huang, R. Zeng, R. Z. Qi, W. Li, and Y. Liu, "Leptin contributes to the adaptive responses of mice to high-fat diet intake through suppressing the lipogenic pathway," PloS one, vol. 4, no. 9, pp. e6884, 2009.

[16] M. C. Hsu, M. E. Wang, Y. F. Jiang, H. C. Liu, Y. C. Chen, and C. H. Chiu, "Long-term feeding of high-fat plus high-fructose diet induces isolated impaired glucose tolerance and skeletal muscle insulin resistance in miniature pigs," Diabetol. Metab. Syndr., vol. 9, no. 1, pp. 81, 2017.

[17] D. Sánchez, B. Muguerza, L. Moulay, R. Hernández, M. Miguel, and A. Aleixandre, "Highly methoxylated pectin improves insulin resistance and other cardiometabolic risk factors in Zucker fatty rats," J. Agric. Food Chem., vol. 56, no. 10, pp. 3574-3581, 2008.

[18] N. Arias, M. T. Macarulla, L. Aguirre, M. G. Martinez-Castano, and M. P. Portillo, "Quercetin can reduce insulin resistance without decreasing adipose tissue and skeletal muscle fat accumulation," Genes Nutr., vol. 9, no. 1, pp. 361, Jan, 2014.

[19] N. Kamalakkannan, and P. S. Prince, "Antihyperglycaemic and antioxidant effect of rutin, a polyphenolic flavonoid, in streptozotocin-induced diabetic wistar rats," Basic Clin. Pharmacol. Toxicol., vol. 98, no. 1, pp. 97-103, Jan, 2006.

[20] B. Sutariya, and M. Saraf, "Betanin, isolated from fruits of Opuntia elatior Mill attenuates renal fibrosis in diabetic rats through regulating oxidative stress and TGF- $\beta$ pathway," $J$. Ethnopharmacol., vol. 198, pp. 432-443, 2017.

[21] I. Dhananjayan, S. Kathiroli, S. Subramani, and V. Veerasamy, "Ameliorating effect of betanin, a natural chromoalkaloid by modulating hepatic carbohydrate metabolic enzyme activities and glycogen content in streptozotocin - nicotinamide induced experimental rats," Biomed. Pharmacother., vol. 88, pp. 1069-1079, Apr, 2017. 